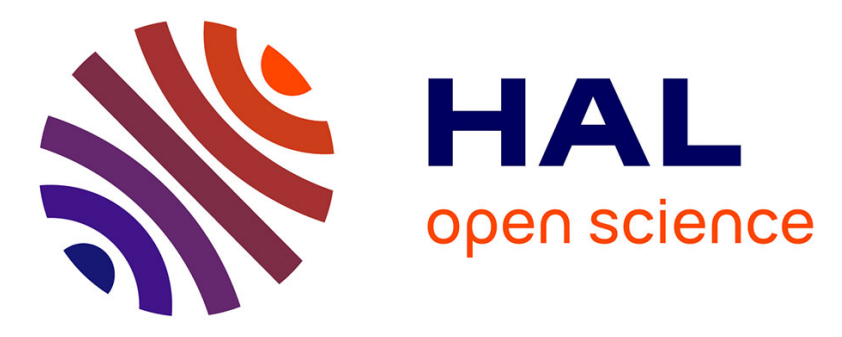

\title{
Flexibility of Thiel's embalmed cadavers: the explanation is probably in the muscles
}

\author{
Mehdi Benkhadra, André Bouchot, Julien Gérard, Denis Genelot, Pierre \\ Trouilloud, Laurent Martin, Claude Girard, Alain Danino, Friedrich \\ Anderhuber, Georg Feigl
}

\section{To cite this version:}

Mehdi Benkhadra, André Bouchot, Julien Gérard, Denis Genelot, Pierre Trouilloud, et al.. Flexibility of Thiel's embalmed cadavers: the explanation is probably in the muscles. Surgical and Radiologic Anatomy, 2011, 33, pp.365-368. hal-00697264

\section{HAL Id: hal-00697264 \\ https://u-bourgogne.hal.science/hal-00697264}

Submitted on 15 May 2012

HAL is a multi-disciplinary open access archive for the deposit and dissemination of scientific research documents, whether they are published or not. The documents may come from teaching and research institutions in France or abroad, or from public or private research centers.
L'archive ouverte pluridisciplinaire HAL, est destinée au dépôt et à la diffusion de documents scientifiques de niveau recherche, publiés ou non, émanant des établissements d'enseignement et de recherche français ou étrangers, des laboratoires publics ou privés. 


\title{
Flexibility of Thiel's embalmed cadavers: the explanation is probably in the muscles
}

\author{
Mehdi Benkhadra ${ }^{1,2} \square$, André Bouchot ${ }^{3}$, Julien Gérard ${ }^{1}$, Denis Genelot ${ }^{1}$, \\ Pierre Trouilloud ${ }^{1}$, Laurent Martin ${ }^{4}$, Claude Girard ${ }^{2}$, Alain Danino ${ }^{5}$, \\ Friedrich Anderhuber $^{6}$ and Georg Feigl ${ }^{6}$
}

(1) Laboratory of Anatomy/INSERM U887, Faculty of Medicine, Medical University of Burgundy, $7 \mathrm{Bd}$ Jeanne d'Arc, 21033 Dijon, France

(2) Department of Anesthesiology and Intensive Care, Teaching Hospital, Bd Marechal De Lattre de Tassigny, 21034 Dijon, France

(3) Imagery Platform, IFR100, Medical University of Burgundy, 7 Bd Jeanne d'Arc, 21033 Dijon, France

(4) Department of Pathology, Teaching Hospital, Bd Marechal De Lattre de Tassigny, 21034 Dijon, France

(5) Service de Chirurgie Plastique, Hôpital Notre Dame, Centre Hospitalier de l'Université de Montréal, Université de Montréal, 1560 rue de Sherbrooke Est, Montréal, QC, H2L4M1, Canada

(6) Institute of Anatomy, Medizinische Universität, Graz, Austria

\section{$\square$ Mehdi Benkhadra}

Email: $\underline{\text { m.benkhadra@wanadoo.fr }}$

Received: 10 March 2010 Accepted: 5 July 2010 Published online: 15 July 2010

\begin{abstract}
Introduction

The flexibility of cadavers conserved using Thiel's embalming method remains unexplained. We aimed to perform microscopic comparison of muscle and tendon fibres from fresh cadavers (FC), formaldehyde-preserved cadavers (FPC) and cadavers conserved by Thiel's method (TC).

Methods

Muscle and tendon biopsies performed on FC, FPC and TC were conditioned and stained by Masson's trichrome, Sirius red and Ramon y Cajal, then studied under optical microscope. Alignment and integrity of the muscle and tendon fibres were studied.

Results

We observed a modification of the muscle fibres in all specimens from TC, regardless of the type of staining used. The muscle fibres taken from FC and FPC were relatively well conserved, both in terms of alignment and integrity. We did not observe any modification of collagen in either muscle or tendon fibres.

Conclusions

The considerable fragmentation of the muscle proteins, probably caused by certain corrosive chemicals, (e.g. boric acid) present in Thiel's embalming solution, could explain the suppleness of the TC. However, we cannot exclude the possibility of alterations in tendon or muscle collagen, since the experimental methods we used, did not allow for the study of collagen ultrastructure.
\end{abstract}

Keywords Thiel's embalming - Cadavers - Formalin - Flexibility - Histology - Surgical education 


\section{Introduction}

Cadavers conserved using the method described by Thiel [ $7-9]$ are known to present a high degree of suppleness, allowing almost natural mobilisation of various body parts. Several applications in surgery and anesthesiology have been described, and all underline the very life-like characteristics of cadavers preserved with Thiel's method, affording working conditions almost as realistic as in living patients $[\underline{1}-\underline{6}, \underline{11}]$. No explanation for this pronounced suppleness has been put forward to date. We aimed to perform histological comparison of muscle and tendon fibres taken from fresh cadavers (FC), formaldehydepreserved cadavers (FPC) and Thiel's embalmed cadavers, with a view to identifying modifications at tissue level that might explain this mobility.

\section{Methods}

This study was conducted in the Institute of Anatomy, Medical University Graz, Austria, and the Laboratory of Anatomy and Imagery Platform of IFR 100, Dijon, France.

\section{Cadavers}

The cadavers investigated were from persons who had donated their body to scientific research, according to the donation programmes of the two institutes of anatomy involved in this study. Six cadavers were used in our study: two preserved according to Thiel's embalming method, two embalmed with formalin and two unembalmed FC. The FC were obtained less than $24 \mathrm{~h}$ after death.

\section{Materials}

For each cadaver, biopsies were taken from the biceps brachii muscle and brachioradialis tendon. The biopsy was performed with a no. 23 scalpel, longitudinal to the fibre orientation.

\section{Experimental protocol}

The samples fixed by Thiel's method and formalin were dehydrated through progressive increasing concentrations of ethanol, then embedded in paraffin. Conversely, the unfixed fresh samples were embedded directly at $-20^{\circ} \mathrm{C}$ in Tissue Tek Optimal Cutting Temperature (OCT) compound. Samples were sectioned using a microtome for paraffin-embedded samples and a cryostat for the OCT embedded samples.

\section{Staining}

The sections were stained using Masson's trichrome, sirius red and according to the Ramon y Cajal procedure. Masson's trichrome makes it possible to visualize type I collagen in green and keratin in red; Sirius red stains collagen types I and III, while Ramon y Cajal staining makes it possible to visualize tissue morphology. With this latter procedure, collagen shows up blue. 


\section{Comparison criteria}

The sample sections were observed under the optical microscope at different magnifications to evaluate structure and ultrastructure. The parameters retained for comparison were integrity of the collagen fibrils in tendon and muscle tissue, as well as integrity and alignment of muscle fibres.

\section{Results}

As shown in Fig. $1 \mathrm{~d}$ and g, we observed major modification of the integrity and the alignment of muscle fibres in all samples taken from Thiel's embalmed cadavers. These changes were visible regardless of the staining protocol used. The muscle fibres had a cut-up, "minced" appearance, but remained aligned. The conjunctive collagen fibrils were also undisturbed.
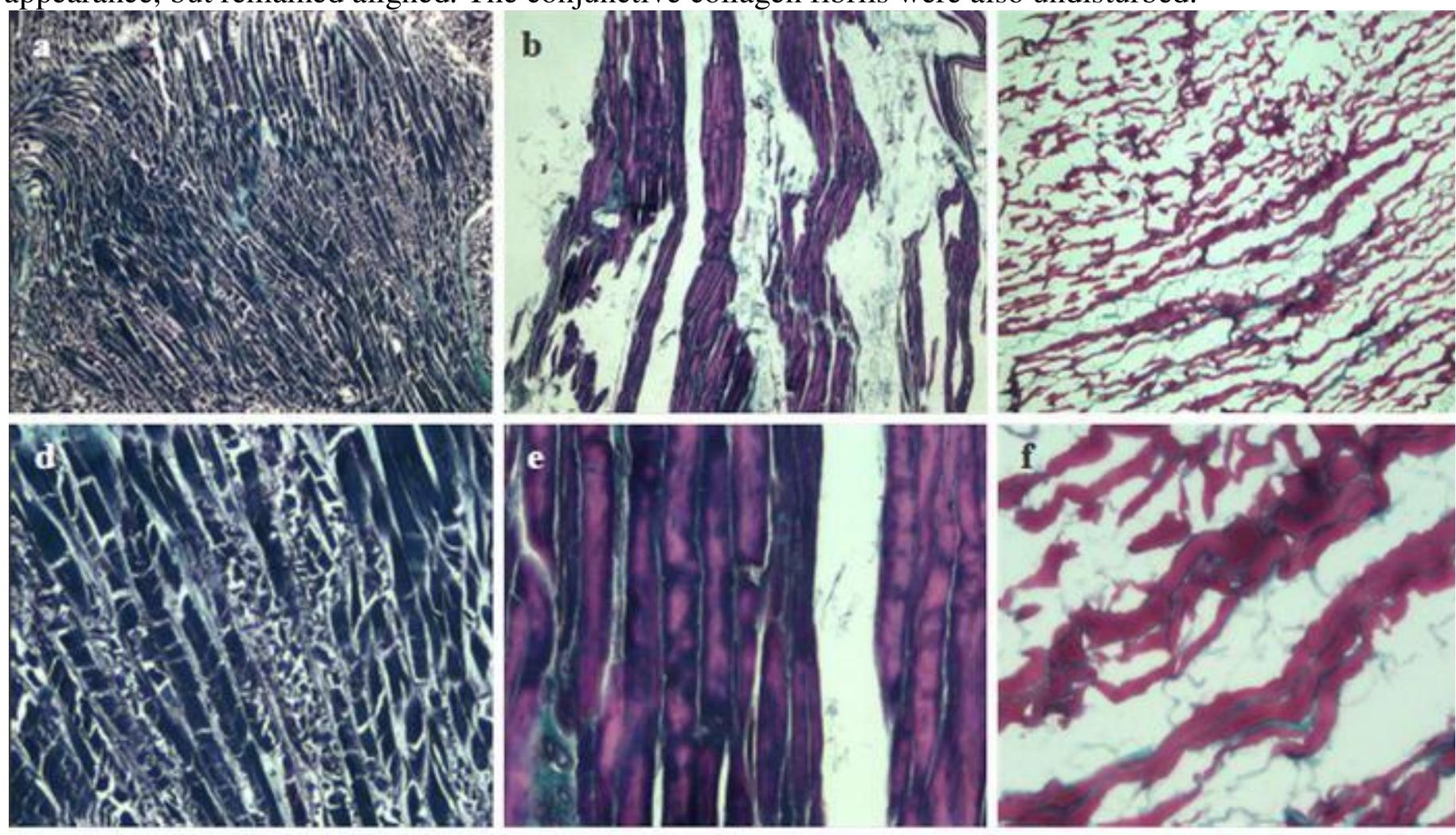

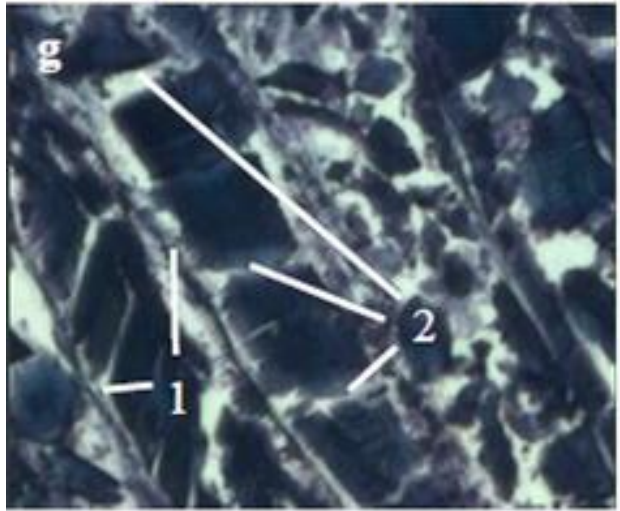

Thiel

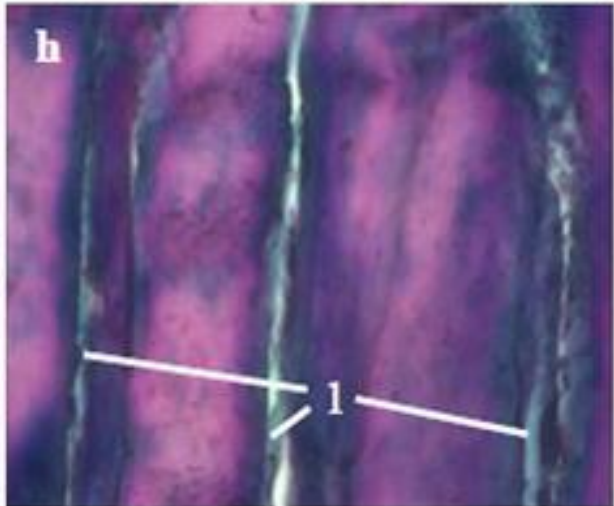

Formalin

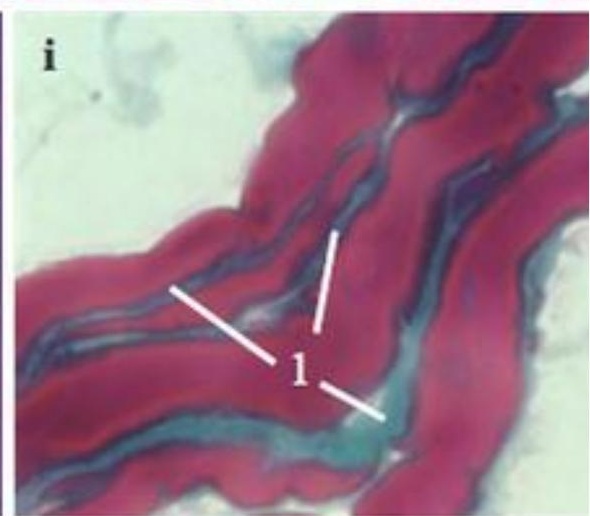

unfixed

Fig. 1 Muscular fibre structure according to fixative product. Masson's trichrome staining of human muscle tissue observed at different magnifications: $\times 2.5(\mathbf{a}, \mathbf{b}, \mathbf{c}), \times 10(\mathbf{d}, \mathbf{e}, \mathbf{f}), \times 40(\mathbf{g}, \mathbf{h}, \mathbf{i})$. Samples 
were fixed with Thiel's solution $(\mathbf{a}, \mathbf{d}, \mathbf{g})$, or formalin $(\mathbf{b}, \mathbf{e}, \mathbf{h})$ and embedded in paraffin, or unfixed $(\mathbf{c}, \mathbf{f}$, i) and embedded in OCT at $-20^{\circ} \mathrm{C}$. 1 Collagen boundary. 2 "Minced" appearance

Figure 1f, $\mathrm{h}$ and i shows that, these changes were not observed in samples from FC or FPC, and indeed, the structure of the fibres from these two sources were quite similar.

No modification of tendon fibres was observed (Fig. 2).
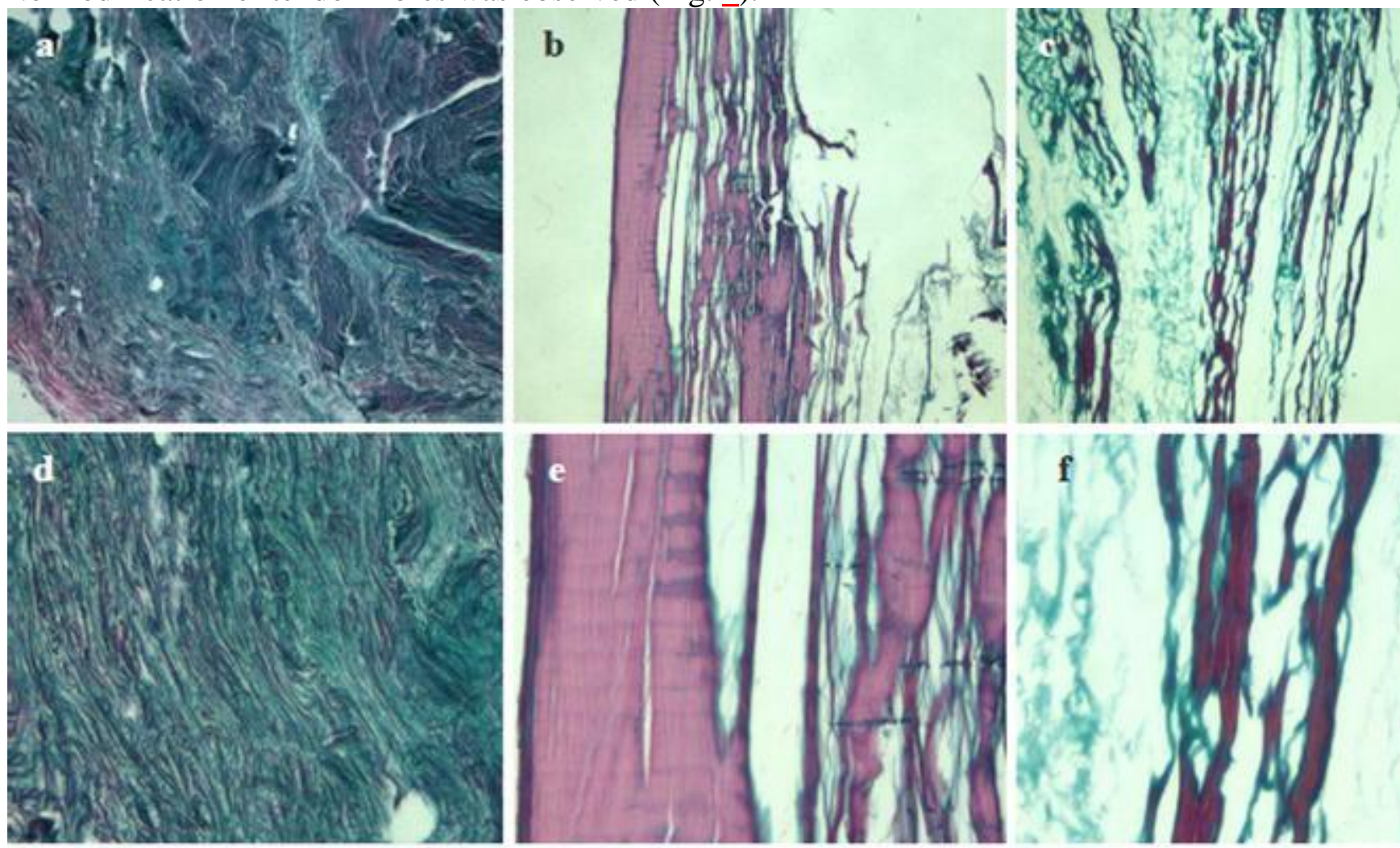

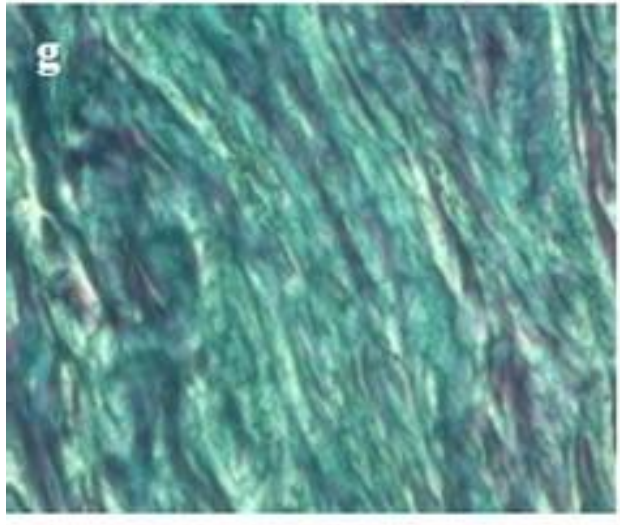

Thiel

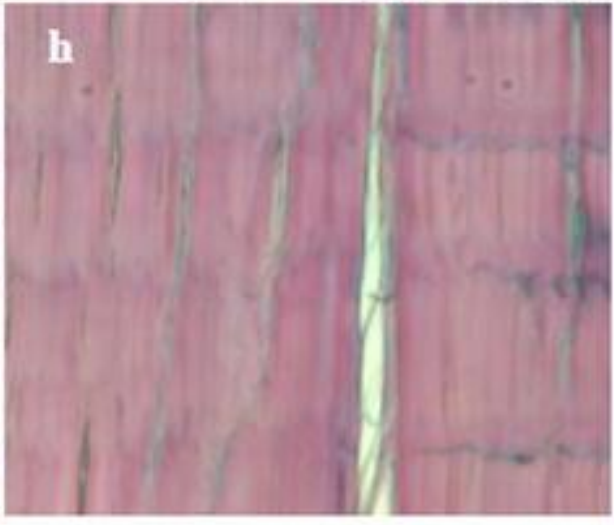

Formalin

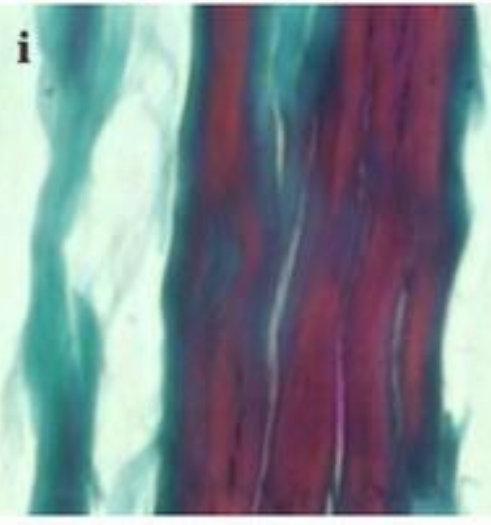

unfixed

Fig. 2 Tendon structure according to fixative product. Masson's trichrome staining of human tendon fibres observed at different magnifications: $\times 2.5(\mathbf{a}, \mathbf{b}, \mathbf{c}), \times 10(\mathbf{d}, \mathbf{e}, \mathbf{f}), \times 40(\mathbf{g}, \mathbf{h}, \mathbf{i})$. Samples were fixed with Thiel's solution (a, $\mathbf{d}, \mathbf{g})$, or formalin $(\mathbf{b}, \mathbf{e}, \mathbf{h})$ and embedded in paraffin, or unfixed $(\mathbf{c}, \mathbf{f}, \mathbf{i})$ and embedded in OCT at $-20^{\circ} \mathrm{C}$ 


\section{Discussion}

The modifications that we observed in the muscle samples from Thiel's embalmed cadavers, could conceivably explain the exceptional suppleness of these cadavers. It appeared that the collagen structure forming the basic muscle structure is preserved. The muscle fibres appeared as if they had been cut up or ground, and no longer had any hold, but were contained in a collagen sheath that remained intact, thus preserving the overall form of the muscle. Acids are well-known to have very corrosive effects on proteins and in our case muscle proteins. The only acid which is present in Thiel's mixture is boric acid, thus we suspected it to be the reason for the observed damage. To our knowledge, the other chemicals of the Thiel's embalming solution could not be involved in this very singular destruction of the muscles [79]. We also cannot exclude the possibility of alterations in collagen ultrastructure. By light microscope examination, we did not observe any such changes, but the collagen structure would need to be studied more closely by electron microscopy. It would be interesting to analyse other tissue types, which also maintain a near natural degree of mobility and plasticity. For example, blood vessels that can be dilated, when pulse is simulated using a balloon [4], peripheral nerves, which are stretched in extension-flexion of the limbs, and certain organs such as the lungs.

The muscular changes induced by Thiel's technique require precautions in the possible applications of this method. Appropriate applications, should be those requiring muscle flexibility, as realistic as possible, such as training in laparoscopic [ $\underline{11}]$, or plastic $[\underline{1}, \underline{10}]$ surgery, airway training (tracheal intubation) [3] and ultrasound regional anesthesia [2]. Applications concerning the precise biomechanical properties of muscle fibres must be considered with caution. A study comparing biomechanical properties of muscles and tendons preserved by Thiel's method, formalin or unfixed, would be very interesting, and also refine the model and precise appropriate and unappropriate uses of Thiel's embalmed cadavers.

\section{Conclusion}

The alterations in muscle fibres that we observed in Thiel's embalmed cadavers could explain the unique suppleness and flexibility of cadavers embalmed by this method. However, alterations in collagen ultrastructure, undetected by our methodology of light microscope examination, could be an alternative explanation.

Acknowledgments We would like to thank Mr Romain Toussaint and Mrs Amandine Chlemaire, preparers of the Imagery Platform of IFR100, for their helpful technical support. We thank Mrs Fiona Ecarnot for translation and editorial assistance. This study was financed by the fund of the laboratory of anatomy, Medical University of Dijon, France. 